\title{
An Analyze of Spatial Newspapers in a Certain Period
}

\author{
Pınar Sarın $^{\mathrm{a},}$ *, Necla Uluğtekin ${ }^{\mathrm{b}}$, \\ ${ }^{a}$ ITU Geomatic Engineering, pinarsarin@gmail.com \\ ${ }^{b}$ ITU Geomatic Engineering, ulugtek@itu.edu.tr \\ * Corresponding author
}

Keywords: Map Use, Journalistic Cartography, Geo-referencing

\begin{abstract}
:
Maps and map-like images are important tools that are often used in written and visual media. Especially, spatial information used in written media have an important role to spread geographic and semantic data to reader. Considering the frequency and ease of access of newspapers to people, the importance of using spatial information in newspapers increases. However, few studies have been conducted on spatial images used in newspapers. These studies, which are few in the literature, were carried out by cartographers such as Monmonier, Pipps and Balchin. Whereas the extent of spatial information that reaches millions of people everyday through newspapers should be explored.

With this concern, the use of spatial images used within the scope of various topics in various sections of the newspapers was investigated. In this context, more than 7000 spatial images of 8 newspapers published within the determined specific time interval (1928-2000) were archived. The time interval for the main research was the Alphabet Revolution of Turkish Republic (1928) and the period when web newspapers were launched (2000). The archive work was carried out through the libraries where these records are located and web archives. Determined items from the newspapers with archive works are classified in a web-based data base. The classifications are designed according to the flow diagram shown in the figure 1.
\end{abstract}

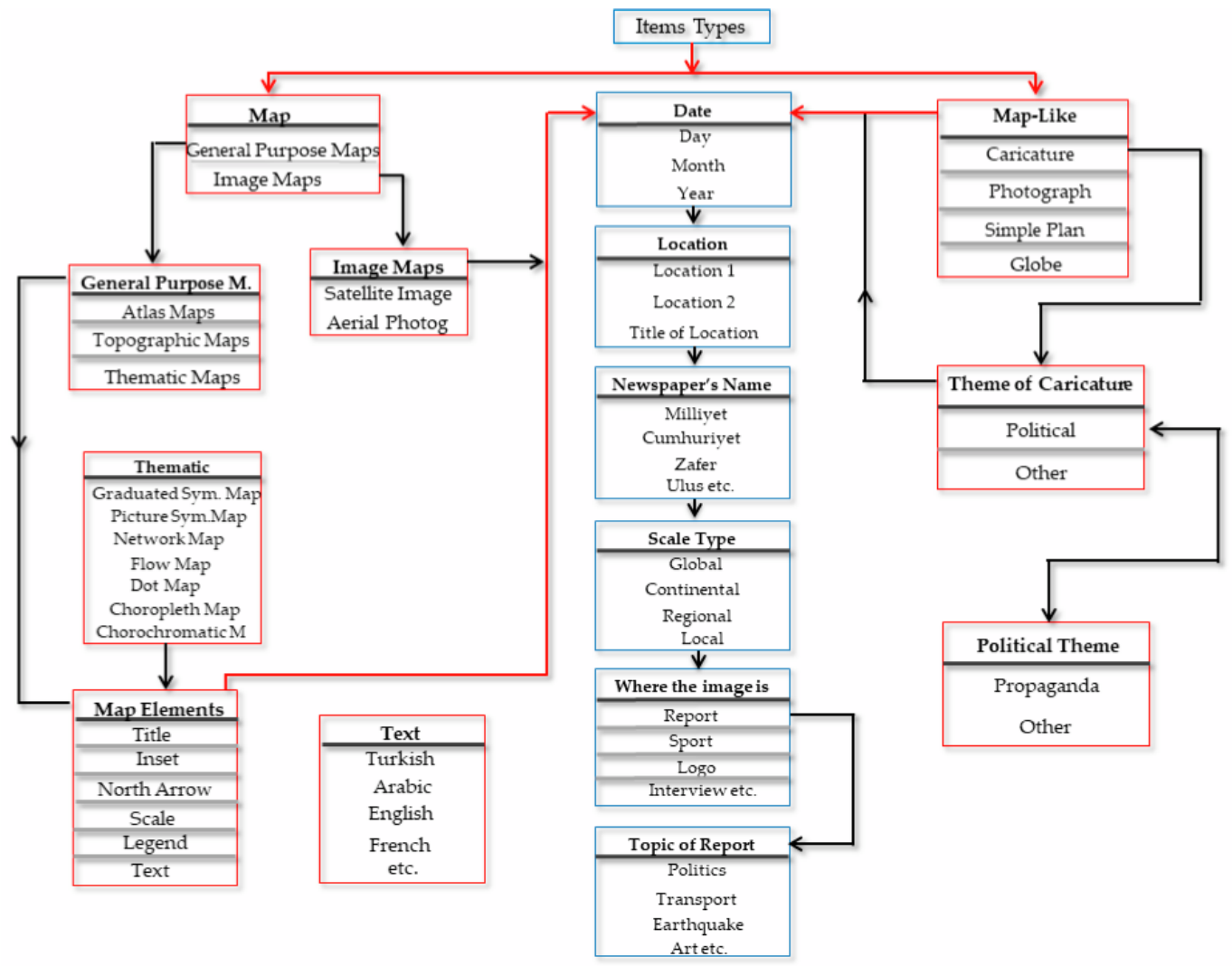

Figure 1. Principle of the Database

As seen in this diagram, the classification includes cartographic and thematic analysis as well as spatial analysis with geo-referencing. These spatial images focused on examined under two main titles as map and maps-like with cartographic scope. After it was determined that the data was map or map-like, other cartographic classifications were made accordingly. For example, if the data is a map, the map type, the map elements and approximate scale of the map are evaluated. Within the scope of the thematic analysis, the section in which each data is contained in the newspaper 
was determined. In addition to this, the subject of spatial information has also been determined. In scope of these analysis, the role of the maps in the newspapers are discussed and the most used subject and newspaper sections of maps were determined. Each data evaluated thematically was also recorded spatially in the database system. According to this system called geo-referencing, each data is recorded on a Google map according to the region it actually represents on the earth. These steps regarding the study were carried out for the following purposes: Collect map and map-like images from selected newspapers in a certain period, classify of the images in newspapers according to their geographic and thematic features to construct a web-based and geo-spatial database to share with researchers, investigate which parts of newspapers the maps are mostly included in, determine the topics and events that increase map usage in the newspapers. In these directions, for this article; thematic analyzes of spatial images in 5 newspapers published between 1970 and 2000 were made. The reason for choosing this historical period for this article is that there are many political events that will increase the use of spatial information in Turkey and its geography for the period. Thematic evaluations of 3452 spatial images of 5 newspapers published during this period will be presented in this study. Within the scope of these analysis, it was questioned in which part of the newspapers more spatial information was needed and in which subject more spatial information was applied. In addition, it has been researched that the use of spatial information increased in the specified historical period, during which years and during which events. Particularly, the use of spatial information during the specified political events will be examined.

This study, which deals with the spatial information roles of newspaper maps in the relevant sections, is one of the few contributions in cartographic literature. It is thought that the study not only enables the questioning and analysis of spatial information within the data range, but also the study will enable machine learning and story map applications with the database query possibilities and wide data opportunity. It is thought that the qualitative analysis achieved with this study can be integrated into the machine learning system with the same cartographic point of view. Similarly, this study, whose data collection and data analysis stages were done manually, can be an inspiration for story map applications with sufficient software infrastructure. The study, which presents a historical evaluation of data journalism in Turkey, is an important analyze of newspaper maps that contribute to geographical education of the society. With the wide data opportunity, it offers, analyzes could be made from many different perspectives. In this context, the study promises different study topics related to the pre-internet and post-internet era. In addition, the study provides many opportunities for multi-disciplinary studies. In this respect, it is foreseen that thousands of data classified on a database will be evaluated within the scope of data driven journalism. 